\title{
Keynote: \\ 10 Years of Semantic Web Research: Searching for Universal Patterns
}

\author{
Frank van Harmelen \\ Vrije Universiteit Amsterdam, NL
}

\begin{abstract}
At 10 years of age, there is little doubt that the Semantic Web is an engineering success, with substantial (and growing) take-up in business, government and media. However, as a scientific field, have we discovered any general principles? Have we uncovered any universal patterns that give us insights into the structure of data, information and knowledge, patterns that are valid beyond the engineering of the Semantic Web in its current form?

If we would build the Semantic Web again, surely some things would end up looking different, but are there things that would end up looking the same, simply because they have to be that way?
\end{abstract}

